\title{
A case study on vibration control in a boring bar using particle damping
}

\author{
M. Senthil kumar ${ }^{1}$, K. M. Mohanasundaram² and B. Sathishkumar². \\ ${ }^{1}$ Department of Mechanical Engineering, PSG College of Technology Coimbatore 641 004, INDIA. \\ ${ }^{2}$ Department of Mechanical Engineering, Jansons Institute of Technology, Coimbatore 641 659, INDIA. \\ Corresponding author: msenthil_kumar@hotmail.com
}

\begin{abstract}
Particle damping offers potential for designing a better passive damping technique with a minimal impact on the strength, stiffness and the weight of a vibrating structure. Due to its conceptual simplicity, potential effectiveness over a wide frequency range, temperature and degradation insensitivity, and cost-effectiveness, particle damping is an attractive passive damping. This paper investigates the efficiency of particle damping in control of vibrations in a boring bar using copper and lead particles of various sizes. Their damping performances were compared and the results are presented.
\end{abstract}

Keywords: particle damping, boring bar, vibration control.

DOI: http://dx.doi.org/10.4314/ijest.v3i8.15

\section{Introduction}

Particle damping is a type of impact damping where multiple particles are placed inside the cavity of vibrating structures. Studies conducted recently have shown the effectiveness and potential application of particle dampers. Particle damping is used to increase structural damping by filling particles in an enclosure attached to the vibrating structure. The unique feature of this type of damping is that high damping is achieved by converting kinetic energy of the structure to heat as opposed to the more conventional methods of damping, where the elastic strain energy stored in the structure is dissipated into heat. The particles absorb kinetic energy of the vibrating structure and convert it into heat through inelastic collisions among the particles and between the particles and the enclosure. It involves the potential of strain energy absorption and dissipation of the same through momentum exchange between moving particles and vibrating walls, friction, impact restitution, and shear deformation.

Particle damping involves energy dissipation by a combination of many factors such as collision, friction and shear damping (Panossian, 1992). A considerable high level of complexity for their modeling and damping analysis is brought in by the particleparticle and particle-wall collisions. Owing to complex interactions involved in particle damping, a comprehensive analytical tool is not in existence yet (Fowler et al., 2000). This technology is a special area and the literature is rather inadequate. The non-linear behavior of particle damping is well associated with two essential mechanisms of damping: friction and impact (Kuamin et al., 2004). In these two phenomena, friction principally depends upon the co-efficient of friction of the particle, whereas impact is a factor of mass and the size of the particle (Xu et al., 2005).

Single mass impact dampers have also been extensively studied on the damping mechanism and the influence of parameters such as mass ratio, particle size, particle/slot clearance, excitation levels and direction of excitation have also been studied (Panossian, 1992). In the single mass system, direct analysis exists for steady state vibration whilst a simple numerical simulation would divulge damping effect in a free vibration (Xu et al, 2005). Riaz et al. (2011) have simulated particle damping under centrifugal loads whereas Bhatti et al. (2009) have studied particle impact damping in two dimensions.

The relative efficiency of friction, impact and shear friction damping mechanisms depends upon a host of parameters such as particle size, volume fraction, frequency and amplitude of excitation (Panossian, 1991). This has been discussed earlier (Xu et al., 2004). It has been suggested that particle damping is more effective at higher frequencies (Xu et al., 2004). Although particle damping is effective for particles of higher density such as lead, Nayfeh et al. (1997) suggested that particle damping can be 
effective for low density granular materials at frequencies high enough to induce standing waves in the granular fill. Xia et al. (2011) studied the application of particle damping for vibration control in brake drum.

This paper investigates of the efficiency of particle damping in a boring bar. Since the transversal type of particle damper has been discussed exhaustively (Popplewell and Smergicil, 1989), the longitudinal hole filled by particles has been selected for the present study. Inside the longitudinal hole of the boring bar, the vibrating particles would practically generate impact forces normal to the surface of the hole and be distributed over the entire area of contact between the particles and the hole. Copper and lead particles of different sizes were used in the study. The damping ratios of copper and lead particles of different sizes were studied. The effect of hole on the natural frequency of the bar was also studied. The effectiveness of particle damping on the vibration control, has been studied.

\section{Particle Characteristics}

In this paper, the diameter of the used particles exceed at least $20 \%$ of the hole size (nearly $60 \%$ of the hole size). Copper and lead particles used in this study are shown in Fig.1. Selection of particles in this size range is made to isolate shear friction damping from that of dry friction damping, as dry friction damping is less pronounced where the mass of the particle is higher. Since metal particles of high density such as lead and copper are favorable usually, they have been used in spherical shape in this study. The particles and their sizes considered for implementation in the boring bar are given in Table 1.
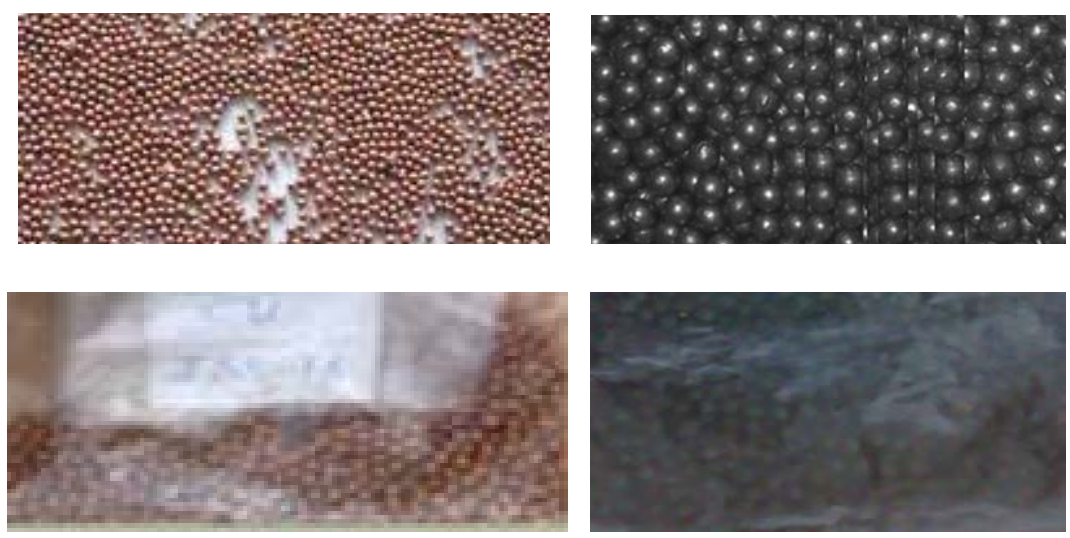

(a) Copper

(b) Lead

Figure 1. Particle samples of different sizes

Table 1. Particles and respective sizes

\begin{tabular}{|c|l|c|}
\hline S.No & \multicolumn{1}{|c|}{ Particle with their sizes } & Density $\left(\mathrm{g} / \mathrm{cm}^{3}\right)$ \\
\hline 1 & Copper $-4.75 \mathrm{~mm}$ & \multirow{2}{*}{8.96} \\
\hline 2 & Copper $-2.36 \mathrm{~mm}$ & \multirow{2}{*}{11.34} \\
\hline 3 & Lead $-3.2 \mathrm{~mm}$ & \\
\hline 4 & Lead $-4.75 \mathrm{~mm}$ & \\
\hline
\end{tabular}

\subsection{Volumetric Packing Ratio}

The particles can be filled either fully or partially in the containing holes. Packing ratio is the ratio of volume of filled space to the unfilled volume in the containing holes. It has been suggested in previous works (Xu et al., 2004) that a packing ratio of 1 renders the maximum damping effect. In all the experiments conducted, a packing ratio of 1 is considered.

\section{Experimental Investigations}

\subsection{Structural Dynamic Properties of a Boring Bar}

In a boring operation, the boring bar is subjected to dynamic excitation during a cutting operation. This will introduce a time varying deflection of the boring bar. If the frequency of the excitation coincides with one of the natural frequencies of the boring bar, a condition of resonance is encountered. In order to avoid resonance, the natural frequencies of boring bar were determined.

In the cutting experiments, standard $55^{\circ}$ diagonal carbide grade (TN7015) inserts have been used. These inserts have tool geometry with the ISO code DNMG 150608-SL with chip breaker geometry for medium roughing. The boring bar (Fig.2) used is S40T PDUNR15. Although the natural frequencies for the first three modes of this tool are known (Petterson, 2002), the tool used 
for experimentation here is a modified form where an $8 \mathrm{~mm}$ diameter hole is drilled along the length of the shank as shown in Fig.3. The natural frequencies of this modified tool with properties given in Table 2 have been determined.

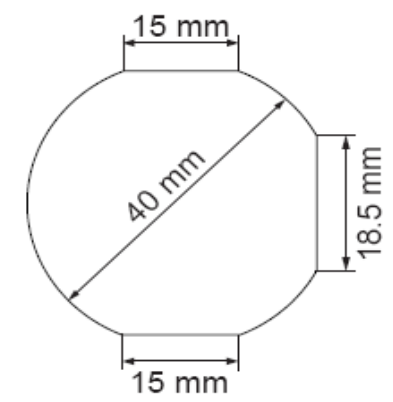

Figure 2. Cross-section of the boring bar

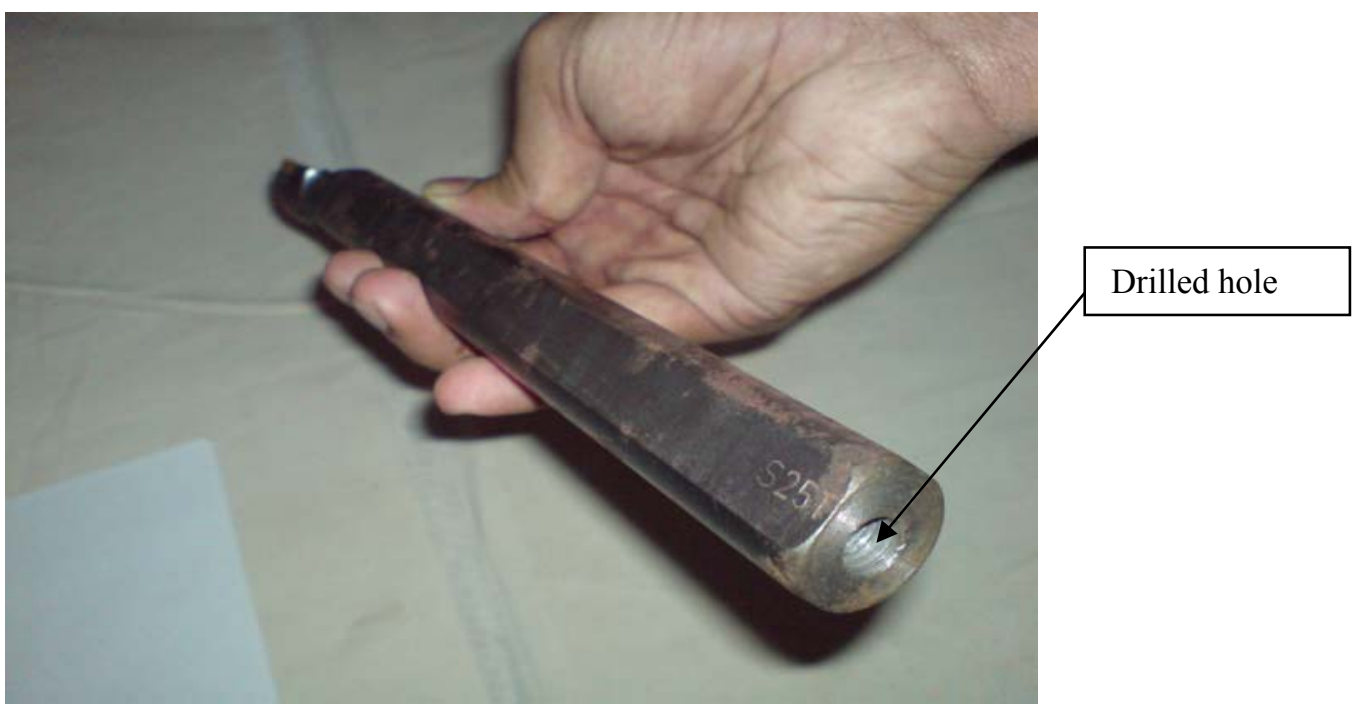

Figure 3. Boring tool with drilled hole

Table 2. Values used in theoretical Euler-Bernoulli calculations

\begin{tabular}{|l|l|l|}
\hline Variable & Value & Unit \\
\hline E, Modulus of elasticity & $207 \times 10^{9}$ & $\mathrm{~N} / \mathrm{m}^{2}$ \\
\hline L, Length of the bar & 0.2 & $\mathrm{M}$ \\
\hline I, Moment of inertia & $1.245 \times 10^{-7}$ & $\mathrm{~m}^{4}$ \\
\hline$\rho$, Density & 7800 & $\mathrm{~kg} / \mathrm{m}^{3}$ \\
\hline A, Cross sectional area & $1.2386 \times 10^{-3}$ & $\mathrm{~m}^{2}$ \\
\hline
\end{tabular}

In order to calculate the natural frequencies of this modified tool, the Euler-Bernoulli equation (Eq.1) of the beam (Thomson and Dahleh, 2007) has been used. The fundamental natural frequency of ' $\mathrm{n}$ ' ' mode (in $\mathrm{Hz}$ ) is given by,

$$
f_{n}=\frac{1}{2 \pi}\left[\beta_{n} l\right]^{2} \sqrt{\frac{E I}{m l^{4}}}
$$

where $\mathrm{m}$ is the mass density of beam per unit length and the constant, $\beta_{\mathrm{n}} 1$ for the first three natural frequencies for clamped -free beam are 1.8752, 4.6941 and 7.8548. It has been reported that the natural frequency of the unmodified boring bar is around 600 $\mathrm{Hz}$ (Petterson, 2002). The natural frequencies of the modified boring bar are calculated to be around $723 \mathrm{~Hz}$ theoretically in the first mode and $4526 \mathrm{~Hz}$ in the second mode.

\subsection{Experimentation}


The PXI 1042-Q (National Instruments, USA) is an interfacing unit designed to act as a data acquisition device, wherein it receives signals from the accelerometer, amplifies it, reduces noise and then transmits the signals to the LabView software, where the acceleration signals can be manipulated and plotted. The boring bar is excited to a particular level of displacement and the vibration is picked up by the accelerometer pickup placed at a distance of $110 \mathrm{~mm}$ from the tip of the insert. The signal from the accelerometer pickup is interfaced with NI PXI-1042 Q and the Lab VIEW program is used to record the signal and obtain the displacement-time plot. Since the influence that the different particles exert on the settling time will have a direct impact on the damping efficiency of the particles under actual machining conditions, settling time is an attribute of a boring bar that ought to be considered while implementing particle damping. An accelerometer of sensitivity $10.2 \mathrm{mV} / \mathrm{g}$ has been used for vibration pick-up. The experiment is repeated with different particles filled in the boring bar and the particles showing the least settling time is considered to be the most effective damping agent. Fig.4 shows the experimental set-up.

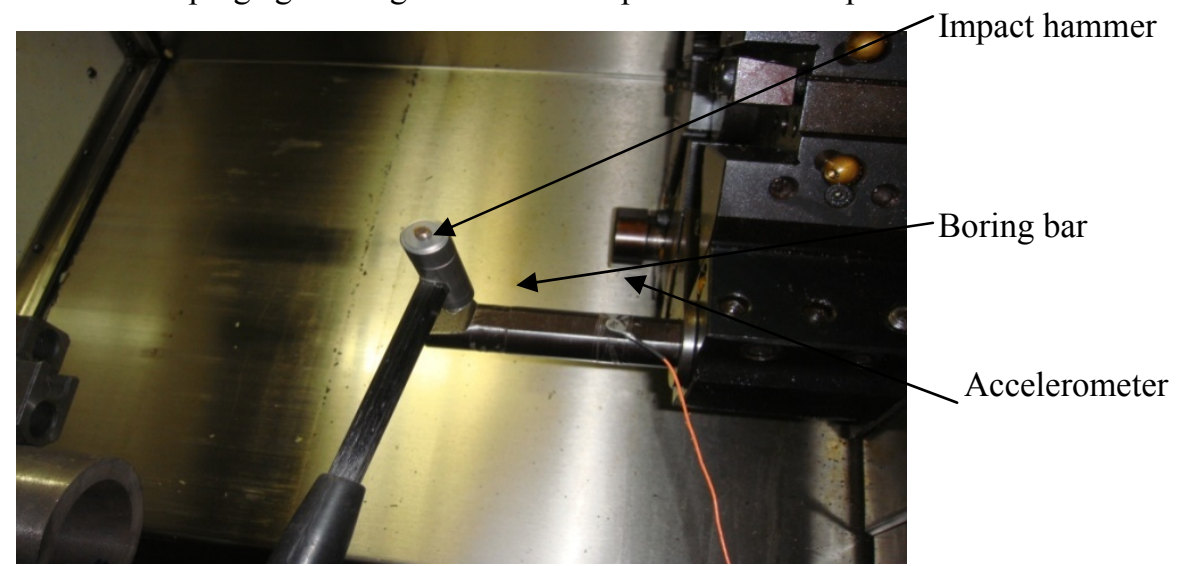

Figure 4. Experiment set-up for settling time using impact hammer

A comparison of the actual frequency values of the solid beam and drilled beam are provided in Table 3 . The natural frequency for the solid beam varies a little from that of the drilled beam. This can be attributed to the fact that the mass and thereby the stiffness of the solid beam changes when it is drilled.

Table 3. Natural frequency comparison between Solid Beam and Drilled beam

\begin{tabular}{|c|c|c|c|c|c|c|}
\hline $\begin{array}{l}\text { Modal } \\
\text { Frequency (Hz) }\end{array}$ & $\begin{array}{l}1^{\text {st }} \\
\text { Mode }\end{array}$ & $\begin{array}{l}2^{\text {nd }} \\
\text { Mode }\end{array}$ & \begin{tabular}{|l|}
$3^{\text {rd }}$ \\
Mode \\
\end{tabular} & $\begin{array}{l}4^{\text {th }} \\
\text { Mode }\end{array}$ & \begin{tabular}{|l|}
$5^{\text {th }}$ \\
Mode \\
\end{tabular} & $\begin{array}{l}6^{\text {th }} \\
\text { Mode }\end{array}$ \\
\hline Solid Beam & 320 & 850 & 1425 & 2800 & 3400 & 4260 \\
\hline Drilled Beam & 340 & 950 & 1815 & 2900 & 3350 & 4245 \\
\hline
\end{tabular}

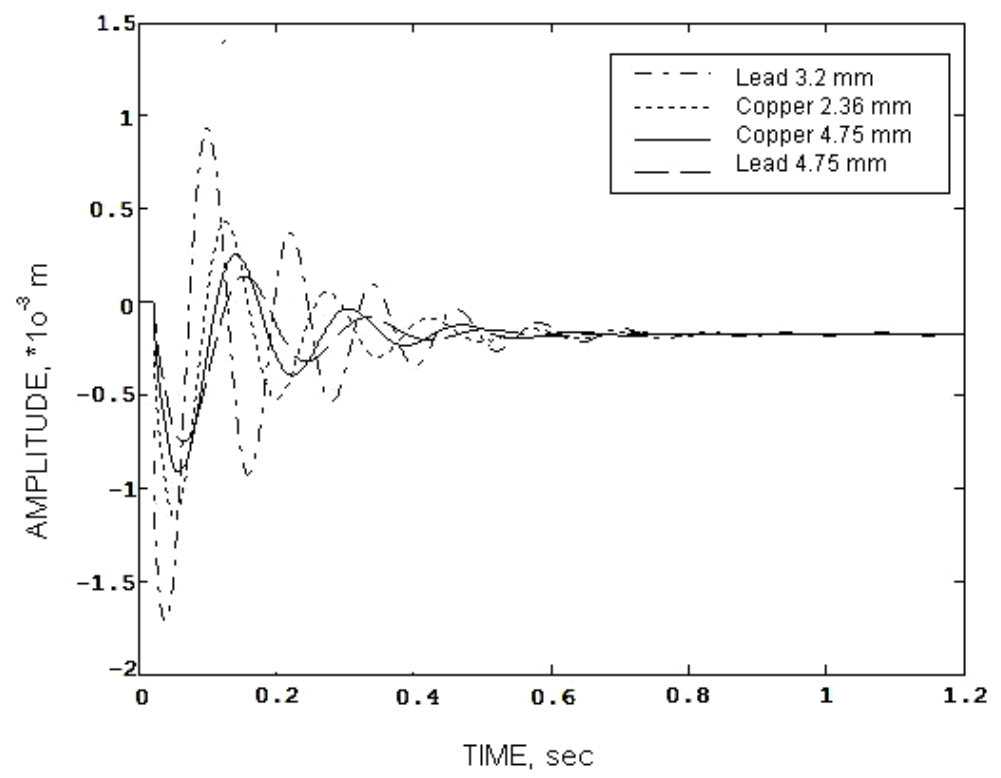

Figure.5 Response of free vibration of boring bar filled with particles 
The slender boring operation was carried out in a CNC turning center, MIDAS 6, at the PSG CNC Training center, PSG College of Technology. The work piece used for the slender boring operation is a cast iron hollow cylinder with an outer diameter of $80 \mathrm{~mm}$, an inner diameter of $60 \mathrm{~mm}$ and a length of $80 \mathrm{~mm}$. The work pieces were cast and pre-machined the required specifications before the slender boring operations were commenced. The damping ratio provided by various particles were found by Eq.(2), (Thomson and Dahleh, 2007). The Table 4 shows the settling time and damping ratio values obtained by the different particles used.

$$
\delta=\frac{1}{n} \ln \left[\frac{x_{0}}{x_{n}}\right]=\frac{2 \pi \varepsilon}{\sqrt{1-\varepsilon^{2}}}
$$

where $\delta$ is the logarithmic decrement, $\mathrm{x}_{0}$ is the initial amplitude, $\mathrm{x}_{\mathrm{n}}$ is the amplitude after ' $\mathrm{n}$ ' cycles have elapsed, and $\varepsilon$ is the damping ratio. From Fig.5, the damping ratios were obtained by using Eq.2 for different particles.

Table 4. Settling time of the boring bar for each particle

\begin{tabular}{|c|c|c|}
\hline Particle & Settling time (s) & Damping ratio \\
\hline Copper $4.75 \mathrm{~mm}$ & 0.95 & 0.086 \\
\hline Copper $2.36 \mathrm{~mm}$ & 1.23 & 0.074 \\
\hline Lead $3.2 \mathrm{~mm}$ & 1.34 & 0.071 \\
\hline Lead $4.75 \mathrm{~mm}$ & 1.15 & 0.085 \\
\hline
\end{tabular}

After the workpiece and tool are setup in the $\mathrm{CNC}$ turning center, the accelerometer pickup is placed at a distance of $110 \mathrm{~mm}$ from the tip of the insert. After the settling time is determined as is mentioned before, a first roughing pass is given to obtain concentricity in the workpiece. The actual experiment is performed during the second roughing pass with a depth of cut $0.6 \mathrm{~mm}$ at $1500 \mathrm{rpm}$ with a feed of $0.15 \mathrm{~mm} / \mathrm{rev}$. The experiment is carried in the same manner for the various particles chosen for the experiment. The displacement - time plot is obtained in the same manner as in settling time determination. The experiment is performed during the roughing pass as opposed to the finishing pass since the vibrations are more pronounced in the roughing pass. The reason for performing the experiment during the roughing operation could be attributed to the fact that particle damping may be implemented in areas where controlling the surface finish is more important than obtaining a higher degree of surface finish. Furthermore, since other finishing operations usually follow a boring operation, obtaining a higher degree of surface is not the major concern. Rather controlling the surface is paramount here.

\section{Results and Discussion}

The experiments on boring bar held as a cantilever were carried out using 4 different particles with 10 trials for each particle and the most repeated result was taken into account. The experiments were conducted to determine the settling time for the boring bar filled to $100 \%$ capacity with different particles. The settling time was determined by giving an impact pulse to the bar using an impact hammer. The boring bar was used in the application study for determining the set of particles yielding the best result.

It was observed that settling time increases with decrease in particle size. Theoretical results (Xu et al., 2004) indicate that, as the particle size decreases, frictional shear should also increase thus increasing the damping effect. In this case, since both particles are high density materials and of larger sizes this may not be true. The larger mass in this condition may work to increase the efficiency of impact damping, thus resulting in a descending trend for impact damping with increasing particle size. The displacement plots of the boring bar used without particles are superimposed with the displacement plots of the boring bar with various particles filled upto $100 \%$ volumetric packing ratio and are shown in Figs. 6,7,8 and 9.

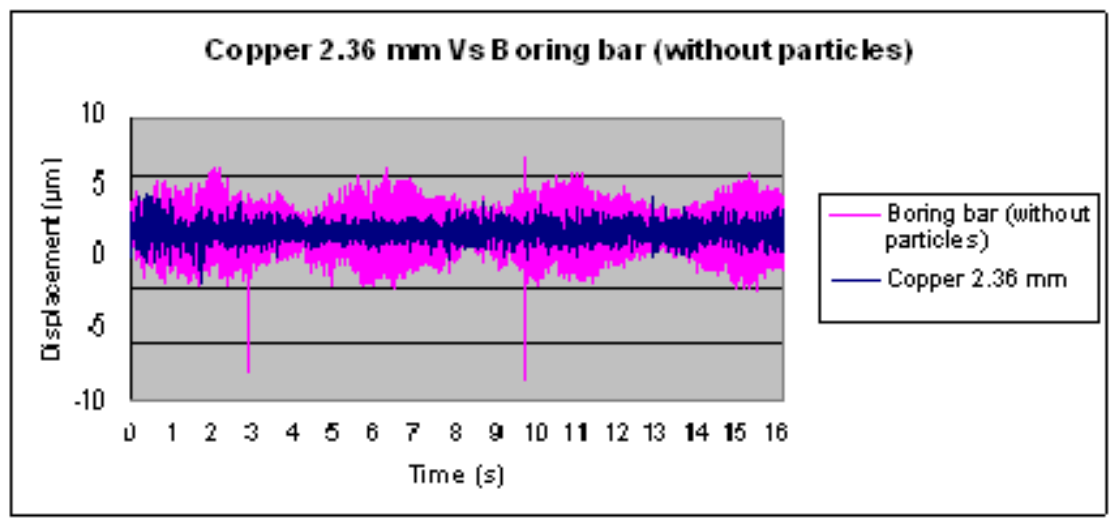


Figure 6. Displacement Vs Time for Copper $2.36 \mathrm{~mm}$

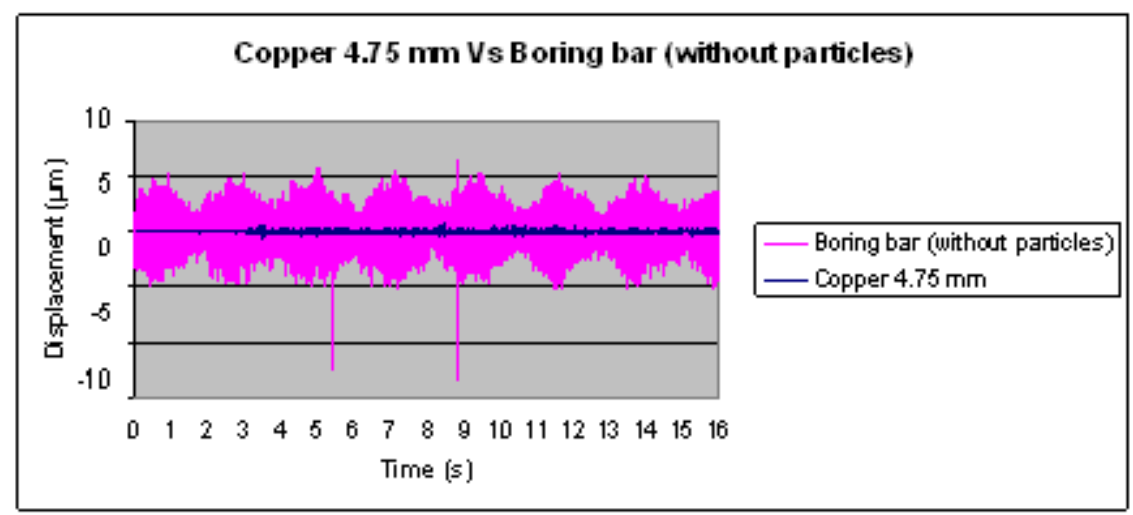

Figure 7. Displacement Vs Time for Copper $4.75 \mathrm{~mm}$

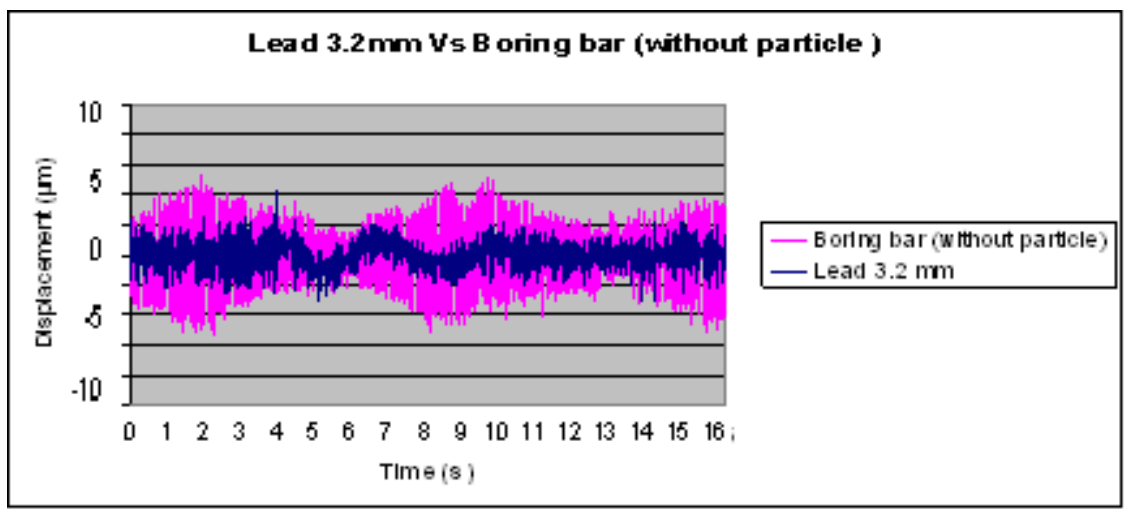

Figure 8. Displacement Vs Time for Lead $3.2 \mathrm{~mm}$

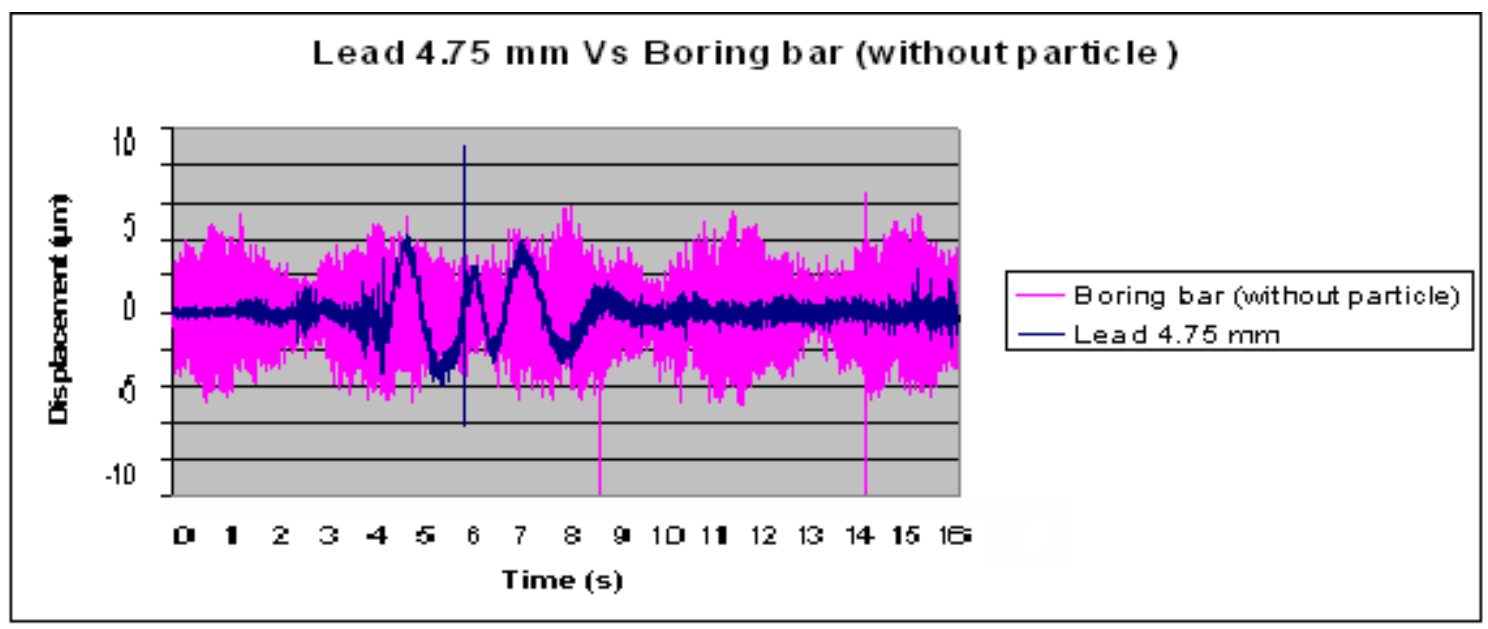

Figure 9. Displacement Vs Time for Lead $4.75 \mathrm{~mm}$

It can be observed from Figs. 6-9 that the damping performances of copper particles follow the same trend as that in settling time. Copper $2.36 \mathrm{~mm}$ and Copper $4.75 \mathrm{~mm}$ show a maximum reduction of vibration by about $80 \%$ and $90 \%$ respectively. This trend concurs with that found in the settling time of the boring bar for the respective particles. Although the exact reason for this trend is hazy, it is possible to infer to a certain degree that as the size of the particle increases, impact damping takes over as the primary damping mechanism. 
It has already been observed that copper $4.75 \mathrm{~mm}$ is a better damper than lead 4.75 , this is again reflected in the results shown in Figs.8-9. Lead $3.2 \mathrm{~mm}$ and $4.75 \mathrm{~mm}$ show a maximum reduction of about $65 \%$ and $81 \%$ which are lesser than that exhibited by copper particles in the same size range. From the results, it can be inferred that particle damping is also effective at lower frequencies, given the arrangement allows for greater deflection caused by vibration in the vibrating structure, such as the cantilever arrangement. It was observed that the settling increasing with decrease in particle size. The results indicate that as the particle size decreases, frictional shear should also increase thus increasing the damping effect. The larger mass in this condition may work to increase the efficiency of impact damping, results in a descending trend for impact damping with increasing particle size. The damping performances of copper particles follow the same trend as that in settling time. This trend concurs with that found in the settling time of the boring bar for the respective particles.

The damping property of copper shots is highest in the frequency of $340 \mathrm{~Hz}$ wherein the reduction in displacement is close to 66 $\%$. For both copper and lead particles the damping performance at $340 \mathrm{~Hz}$ is much higher than that recorded for a freely suspended beam. This phenomenon of higher particle damping performance for lower frequency ranges in a cantilever arrangement can be attributed to the copper's high internal coefficient of friction, and to the fact that longitudinal shear gradient is high due to higher deflection when the beam is held as a cantilever and forced vibrations are induced. This may tend to cause a marked increase in shear friction damping and hence the significant damping effect at this frequency. However, copper's damping capacity is lesser in the range of $950 \mathrm{~Hz}$ and $1815 \mathrm{~Hz}$ although a $35-40 \%$ reduction in displacement is still evident. The lowest \% reduction in displacement is found in the frequency range of $4245 \mathrm{~Hz}$. This trend gives us an indication that shear friction damping may be largely influenced by the frequency of excitation which in turn influences the longitudinal friction gradient.

It was observed that the settling increasing with decrease in particle size. Theoretical results indicate that, as the particle size decreases frictional shear should also increase thereby increasing the damping effect. In this case this is not true, since both particles are high density materials and of larger size and also since the efficiency of impact damping increases with size of the particles. The larger mass in this condition may work to increase the efficiency of impact damping, results in a descending trend for impact damping with increasing particle size. The damping performances of copper particles follow the same trend as that in settling time. Copper $2.36 \mathrm{~mm}$ and Copper $4.75 \mathrm{~mm}$ show a maximum of about $80 \%$ and $90 \%$ respectively. This trend concurs with that found in the settling time of the boring bar for the respective particles. Although the exact reason for this trend is hazy, it is possible to infer to a certain degree that as the size of the particle increases, impact damping takes over as the primary damping mechanism. It has already been observed that copper $4.75 \mathrm{~mm}$ is a better damper than lead 4.75 . Lead $3.2 \mathrm{~mm}$ and $4.75 \mathrm{~mm}$ show a maximum reduction of about $65 \%$ and $81 \%$ which are lesser than that exhibited by copper particles in the same size range.

\section{Conclusions}

The focus of this investigation is on a damping enhancement method with particulate materials. A boring bar is treated with longitudinal holes embedded with metal particles. The experimental investigations on the effectiveness of particle damping in vibration control of boring bar are carried out and reported. The particle damping is found to be remarkably effective. Although it is nonlinear, a strong rate of energy dissipation is achieved within a broadband range. It was found that considerable reduction in vibration was achieved with the particle damping. Results from testing different types of particles indicated that the frequency at which maximum damping occurs and the amount of damping obtained depend on properties of the particles. This suggests that the particles can be designed to target vibration reduction in a specific frequency range.

\section{Acknowledgement}

Author thanks AICTE (All India Council for Technical Education), India for funding this work.

\section{References}

Bhatti, R.A, Wang Y.R, Wang Z.C. 2009. Particle impact damping in two dimensions, Journal of Key Engineering Materials, Vol. 413-414, pp. 415-422.

Fowler B.L., Flint E.M., Olson S.E.. 2000. Effectiveness and predictability of particle damping. Proceedings of the SPIE Conference on Passive Damping and Isolation, Newport Beach, CA, 2000.

Kuanmin Mao, Zhiwei Xu, Michael Yu Wang, Tianning Chen.2004. Simulation and characterization of particle damping in transient vibrations. Journal of Vibration and Acoustics. Vol.126, No.2, pp.202-211.

Nayfeh S.A., Verirame J.M., Varanasi K.K. 1997. Damping of flexural vibration by coupling to low density granular materials. Proceeding of SMIE, USA.

Panossian, H. V. 1992. Structural damping enhancement via non-obstructive particle damping technique. Shock and Vibration Digest, Vol. 1, No. 6, pp. 4-10.

Panossian.H: 1991. Non-obstructive particle damping experience and capabilities. Proceedings of Damping '91 Conference in San Diego, CA, pp. ICB-15, ICV-15, 13-15.

Pettersson L., Vibration analysis of a boring bar, Blekinge Institute of Technology, Research Report No 2002:01. 
Popplewell N, Smergicil S.E., 1989. Performance of bean bag impact damper for sinusoidal external force. Journal of Sound and Vibration. Vol.133, No.2, pp. 193-223.

Riaz A, Bhatti, Wang Yanrong. 2011. Simulation of particle damping under centrifugal loads, International Journal of Aerospace and Mechanical Engineering, Vol. 5, No. 3, pp.165-170.

Thomson, W.T, Dahleh M.D. 2007. Theory of vibrations with applications, $5^{\text {th }}$ Edition, Pearson Education Inc., Delhi, India.

Xia Z., Liu X., Shan Y. 2011. Application of particle damping for vibration attenuation in brake drum, International Journal of Vehicle Noise and Vibration. Vol.7 No.2, pp.178-194.

Xu Z., Wang M.Y., Chen T.. 2004. An experimental study of particle damping for beams and plates, Journal of Vibration and Acoustics, Vol.126 No.1, pp.141-148.

Xu Z., Wang M.Y., Chen T.. 2005. Particle damping for passive vibration suppression: numerical modelling and experimental investigation. Journal of Sound and Vibration, Vol. 279, pp.1097-1122.

\section{Biographical notes}

Dr. M. Senthil kumar is an Assistant Professor in the Department of PSG College of Technology, Coimbatore, India. He received B.E. Degree in Mechanical Engineering (1994), M.E. in Engineering Design (1996) and Ph.D. in active suspension system (2008). He has about 14 years of teaching experience and 1 year of industrial experience. He received ISTE award for Best Research in the field of machine design and vibration in 2006. He has also received AICTE career award for particle damping in 2009. His paper has won second prize (worth US \$ 500) in Intl. Conference held at Jordon. His projects have also won many other awards. He has authored 40 journal papers and about 50 conference papers. Also, he is member in many national and international societies of engineering (ISTE, IE, TSI, IAENG). His fields of expertise include vibration control, composites, smart structures, etc.

Dr. K. M. Mohanasundaram completed his B.E. degree in the year 1982 and his M.E in the year 1986. He was awarded Ph.D in the year 1997. He has got more that 25 years of teaching experience, of which he served PSG College of Technology for 20 years and served Karpagam College of Engineering as Principal for 3 years and at present he is the Principal at Jansons Institute of Technology, Coimbatore, India. 5 candidates have completed their Ph.D work under his supervision and at present 5 candidates are pursuing their Ph.D work under him. He has successfully completed 3 funded projects worth almost Rs.10 million. He has to his credit 3 books, 5 journal papers and 30 papers published in various national and international conferences. He was awarded by the Chief Minister of Tamilnadu in the year 2000 for the contribution in the field of Computer Education at schools.

B. Sathishkumar completed his M.E. in the year 2005 and has got 7 years of teaching experience. At present he is Assistant Professor at Jansons Institute of Technology, Coimbatore, India. He is currently doing his $\mathrm{Ph} . \mathrm{D}$ in the area of the "Particle Damping" under the supervision of Dr. K. M. Mohanasundaram. His M.E. thesis, "Particle Damping for Passive Vibration Suppression" won the best project award for the year 2005 at Kongu Engineering College.

Received February 2011

Accepted January 2012

Final acceptance in revised form May 2012 